\title{
Formation and maintenance of the territorial balance in the conditions of high-rise construction in the city
}

\author{
Tatiana Kisel $^{1, *}$ \\ ${ }^{1}$ Moscow State University of Civil Engineering, Yaroslavskoye shosse 26, Moscow, 129337, Russia
}

\begin{abstract}
High-rise construction results in the need of planning of infrastructure facilities, taking into account the increase in loading, as high-rise construction allows to place considerably bigger number of residents in the limited territory. For this purpose it is necessary to estimate the required and actual level of providing the population with each particular type of the facilities of social infrastructure. The compliance of required and actual level of providing can be characterized as the territorial balance, while the discrepancy acts as the territorial imbalance. The article is devoted to the development of such instruments of planning of urban development, which will allow to create the qualitative urban environment, founded on the territorial balances. Namely, it is devoted to the calculation of level of providing the population with the facilities of social infrastructure, to the determination of level of the imbalance in absolute and relative units and also to the ranging of imbalances on urgency of their elimination. The size of the imbalance is of great importance for planning and realization of managerial influences from the executive authorities, operating the city development. In order to determine the urgency of realization of actions for the construction of facilities of social infrastructure it is offered to range the imbalances according to their size, having determined the deviation size from balance, which is so insignificant that it does not demand any managerial influences (it can be characterized as balance) and also the groups of the imbalances, differing in urgency of managerial influences, directed to the decrease and elimination of the revealed imbalance.
\end{abstract}

\section{Introduction}

Modern megalopolises cannot be considered without high-rise construction. Projects of high-rise construction allow to use expensive city land, which is the most valuable resource, in rather compact territories with high efficiency. However, the use of high-rise construction in urban development proves the need of the use of new approaches to management of city development. One of the tasks of management of urban development is the territorial balance, which assumes maximum providing urban population with the necessary infrastructure. During planning the city development providing the population with the necessary infrastructure facilities, such as schools, kindergartens, hospitals and

* Corresponding author: doremi2@,yandex.ru 
clinics, facilities of culture and sport is to be considered. The existence of enough such facilities in comfortable transport availability is the necessary condition of proper activity in the city.

The modern cities and megalopolises develop mainly on the basis of high-rise construction. Among the world leaders in quantity of skyscrapers Gonk Kong, New York, Dubai, Tokyo, Shanghai and Chicago can be named [1], however, high-rise buildings are available in one and all large cities, that is certainly caused by some objective reasons. The development of technologies of construction has allowed to use with high efficiency the most valuable and limited resource - expensive city land. The territorial resource in city borders, especially in the central part or so-called "centres of gravity" (which are the territories with the high transport availability and infrastructure attracting investors, considerable human flows, etc.) is limited. Therefore the cost of such sites can be the most important element of the investments during the implementation of the investment-andconstruction project.

At the same time the location and environment of the site [2], as well as the set land use mode, the mode and level of the taxation and restriction of town-planning character (for example, the instruction on style (for example, respect for historical appearance of the downtown) or the maximum height of the building) [3,4,5] can influence the value and, respectively, the cost of the land. The use of the technologies of high-rise construction allows to construct large volumes of the area in the small territory, compactly placing offices, and commercial areas and also dwellings.

However, the use of high-rise construction in urban development proves the need of the use of new approaches to the management of the city development. One of the tasks of the urban development management is the territorial balance, assuming maximum providing urban population with the necessary infrastructure. Thus, when planning construction, the need of providing the population with the necessary infrastructure facilities, such as schools, kindergartens, hospitals and clinics, facilities of culture and sport has to be considered. The existence of enough such facilities in comfortable transport availability is the necessary condition of proper activity in the city. Certainly, high-rise construction results in the need of planning of infrastructure facilities, taking into account the increase in loading as high-rise construction, allows to place considerably bigger number of residents in the limited territory. The methodical approach, allowing to form territorial balance between the residential real estate and social infrastructure in the conditions of the use of high-rise construction in urban development is offered in this article.

\section{Materials and Methods}

The research is based on some methodological prerequisites. So, the major and them is the use of provisions of the system approach, within which the modern city is considered as the elaborate system $[6,7,8]$, functioning of the city as whole depends on functioning of its separate parts.

Normal functioning of the city supposses that its basic elements have to be combined: the residential real estate (that is the place of residence of citizens), production and commercial infrastructure (work places of application for residents, existence of the significant amount of places of application of work also forms the city as the main "point of attraction" for the population), transport infrastructure (it creates traficability around the city, which is the most important element of city infrastructure providing convenience of movement of citizens and also necessary for normal functioning of production and commercial infrastructure), engineering infrastructure (it is necessary for normal and comfortable functioning of the modern city and includes all the complex of engineering support by water, energy, withdrawal of drains, waste disposal, etc.), institutional 
infrastructure (it consists of the institutes organizations designed to provide the most effective development of economy: financial and bank structures, analytical and advice centers and also the headquarters of corporations and also the scientific and design organizations, public, political, cultural and other centers, mass media and also other economic and public institutes) and also social infrastructure (it includes objects of education, health care, trade and public catering, consumer services, sport, culture and art). As in any system, the failure in the work of one of the elements (this or that type of infrastructure) will lead to the failure in work of all the system, however in this research special attention is paid to the territorial balancing of the city in relation to the facilities of social infrastructure.

Formation of the balanced infrastructure is one of the most important tasks of management of the city. Respectively, this research is based on accounting of the main functions of management - planning, organization, coordination and control, however the main attention is focused on planning as at the stage of planning of urban development the needs for the facilities of social infrastructure have to be defined. These requirements have to be considered during the planning and placement of the facilities in the territory of the city: enough schools, clinics and hospitals, sport facilities, etc. have to be placed in availability in the territory of the city. In this research the tools, allowing to improve processes of city territorial planning for formation of territorial balance are offered.

As the tools of formation of territorial balance are offered in the research, the obligatory use of the balance method is supposed $[9,10,11]$. The balance method is one of the main methods of planning, which consists in detection of compliance (or discrepancies) and ensuring the balance in any indicators. In the conditions, when we need the solution of the main objective of economy - the satisfaction of public needs for conditions of limited resources, it is possible to consider this method to be the major. It fully belongs to the territorial planning, which main objective is the creation of conditions for the balanced and sustainable development of the city. The balance model developed on the basis of application of this method will allow to reveal discrepancy between existence of different facilities of social infrastructure and the real needs of the population, and also to determine the volume of financial resources, necessary for elimination of territorial disproportions.

The balance method (balance approach) is one of the traditional and most widespread methods of planning, used in the economy. It allows to carry out the comparison of requirements with the resources (available or perspective as well). Thus, the essence of the balance method consists in the identification and the quantitative characteristic of the relations with the parties of any activity, which counterbalance (or have to counterbalance) each other. This method is applied for the wide range of tasks, including balances of fixed assets and production capacities, work and labor, financial, transport, fuel, etc. are developed that in turn means that balances are subdivided on cost (expressed in cost indexes) and natural and grocery (expressed in physical units).

The study of theoretical questions of application of the balance method proved that, due to the universality, balance models are applicable to all the types of systems. The balance method has broad application at various levels and in the different countries $[12,13,14$, 15]. The balance method has universal character, and balance models created on its basis serve as the main instrument of maintenance of proportions in the national economy.

\section{Results}

As one of the most important tasks of the city development management is the formation of territorial balance, it is necessary to present its definition.

The territorial balance is understood as the compliance of qualitative and quantitative characteristics (structure and dimensional indicators) construction projects of various 
functional purpose within the concrete spatial fragment of the territory of the city of the established requirement. This balance represents the actual results of the spatial organization of city-planning activity and is a special case of application of the balance approach to management.

Thus, concrete expression of the territorial balance should be considered as the compliance of the actual and standard indicators on providing the population with housingand-social facilities. In turn, the divergence of these indicators (that is, the imbalance) defines priorities of the construction activity in the concrete territory.

The distribution and practical use of model of territorial balance of the city is the relevant task, having great practical importance and allowing to realize those theoretical and methodical approaches, which conform to modern requirements to development of the urban environment.

It should be noted, that the special importance of formation of territorial balance on the basis of the facilities of social infrastructure. The standard purpose of the urban policy at the moment is formations of the comfortable environment of activity. Such environment is formed, certainly, not only at the expense of the facilities of social infrastructure, but also at the expense of recreational zones, quality of gardening, the qualitative transport system. However, the facilities of social infrastructure create the framework of the urban environment as they provide the population with the facilities, without which any normal activity of the population is impossible. Thus, it is possible to conclude, that the greatest possible providing the residents with the facilities of social infrastructure forms the basis of the comfortable living environment. At the same time the most important task of management is the decrease in the revealed territorial imbalances to their elimination (in case of existence of such opportunity):

$$
L_{M}=\sum_{i=1}^{n} \pm \Delta_{i} \rightarrow \min ,
$$

Where $L_{M}$ - criterion function of management,

$\Delta \mathrm{I}$ - the deviation of the existing providing the population with the facilities of social infrastructure from the requirement

At the same time the deviation of the existing providing the population with the facilities of social infrastructure from the requirement is determined by some factors:

1) the structure of the population (the age structure of the population determines the requirement in number of school places and the average need for clinics, hospitals and other facilities of social infrastructure);

2) the level and structure of birth rate determines the needs for the main facilities of social infrastructure for the medium and long-term outlook;

3) when calculating the existing providing the population with the facilities of social infrastructure it is necessary to consider both the state, and private facilities of social infrastructure.

The territorial balance has to be used in 2 cases:

- when planning complex building in the territory, when there is an opportunity initially qualitatively and in the balanced way to plan the territory;

- within the developed construction when during the identification of territorial imbalances, that is excesses of need for the facilities of social infrastructure over their real quantity, there is the possibility of application of the correcting influences in the city management, that is planning and realization of the actions of urban policy directed construction new or expansion of power of the existing facilities of social infrastructure.

In the first case application of the balance method does not cause any problems regardless of that, low or multy-storied building is planned to be constructed in this territory. Absolutely other administrative situation is formed in case within the developed 
construction investment and construction projects of multy-storied construction which can lead to considerable change of a ratio of the residential real estate and the facilities of social infrastructure on the concrete territory are implemented. For prevention of the situation of emergence of serious imbalances, that is the shortage of the facilities of social infrastructure, during the implementation of projects of high-rise construction it is necessary to carry out the analysis of planned level of providing the population of this area with each type of social infrastructure.

Each of types of social infrastructure, generalized by expression of level of providing (LP soc.infr. (i)) expression is the following:

$$
\text { LP soc.infr. (i) = Vsev. (i) / } \sum \text { Npop., }
$$

где Vserv. (i) - the actual volume of the services, provided by a concrete (i-th) type of the facilities of social infrastructure (in the corresponding measuring instruments);

$\sum$ Npop. - total population in the territory of the city or the concrete area.

Data on the actual number of these facilities and also about their power are necessary for the calculation of the indicators reflecting the level of providing with the facilities of social infrastructure (capacity). Providing level ideally has to coincide with the need for such facilities. In this case it is possible to consider the existence of territorial balance.

In case when the level of providing is not equal to the need for the facilities of social infrastructure (on each of types), it is possible to consider the existence of the territorial imbalance. At the same time the situation when the level of providing is higher than the requirement, should not lead to urgent managerial influence as in this case the situation of sufficient access, comfortable for the population, to necessary social services is provided. The shortage of the facilities of social infrastructure, that is the excess of need for the facilities of social infrastructure over the actual level of providing, on the contrary, proves the need of realization of actions of the urban policy, which are connected with the construction of new facilities of social infrastructure or expansion of already available. The lack of managerial influences can lead to some considerable decrease in the level of comfort and social service for the residents and will reduce the quality of the urban environment, that can also negatively affect the quality of the residents life.

There is a number of the editions, publishing the international ratings of the cities of the world according to the level of quality of life (for example, analytical division of the The Economist magazine, The Monocle magazine), regardless of methodology of the research and quantity of the indicators measured by them it is possible to allocate main units of indicators for the standard of living in the cities:

- political, social and economic situation;

- health care;

- educational opportunities;

- infrastructure;

- possibilities of carrying out leisure in the city (in the range from restaurants, theatres, cinemas and sporting events);

- existence of consumer goods (from food to cars);

- ecological aspects (from the number of parks to air pollution level).

Prevention and the maximum decrease in imbalances is the task of governing bodies of the city.

As the decrease in imbalances in territorial development is one of main objectives of the city management, which realization leads to formation of the comfortable and balanced territory of the city needs to be defined more precisely concepts of the territorial imbalance. Previously it is necessary to mention that the imbalance can be understood in absolute and relative expression. 
The relative imbalance (Imb.rel.) is the degree of divergence of the need for providing social infrastructure with the facilities, which for the convenience (in relative expression, per 1000 people, and the corresponding actual indicators. Thus, Imb.rel. represents the deviation from the requirement, revealed on the basis of demographic data. For example, the need for providing with $i$-th facility of social infrastructure makes 0,15 . The actual providing makes 0,11 . Then there is Imb.rel. $=0,04$;

The absolute imbalance (Imb.abs.) is the divergence between the need for providing and the actual indicators of providing with the facilities of social infrastructure, expressed in physical units. For example, the absolute imbalance (Imb.abs.) is determined by the ratio:

$$
\text { Imb.abs. = Imb.rel. } \times \text { Qpop. / } 1000
$$

where Qpop. - population of the territory (people).

As that was mentioned, the identification of imbalances within the developed building has already been told, gives information on in what territory what types of facilities to be constructed and what size (power, capacity) there have to be these facilities. However within the exfoliating building in relation to territorial balance the main and obvious objective restriction is spatial potential. That is understood as all nominal set of spatial fragments within the territory which can be used for construction. The following main components can be a part of this spatial potential:

1) a reserve of the free areas under building. This reserve in the large cities is, as a rule, extremely insignificant;

2) the areas received as the result of redevelopment of industrial territories;

3) one more component of spatial potential is implementation of actions for updating of areas of the developed building (demolition of old, functionally outdated and dangerous structures and construction in their territory of new objects)

Concerning imbalances, that should be noted the following: imbalances take place in absolute majority of real managerial situations and, depending on their size, not always demand some urgent managerial influences directed to their fastest elimination. So, for example, the situation when the imbalance does not exceed admissible level or its value so can take place a little (for example, makes about 10-3 and less) that in fact you should not consider it as the imbalance from the managerial point of view. Thus, the balance from the managerial point of view is not the exact figure characterizing full compliance between a resource and the need for him, but some interval of values allowing a deviation from balance value. This condition is expressed by a ratio:

$$
\pm \Delta \mathrm{i}\left(\sum \pm \Delta \mathrm{i}\right) \leq \varepsilon
$$

Where $\varepsilon$ is the border of managerial range of deviations.

In other words, there is some range $\pm \Delta \varepsilon$ within which the managerial situation (the condition of the operated process) is recognized as the satisfactory and not demanding the correcting managerial influences. Only when deviations overstep the bounds of the specified range, there is necessary a development and adoption of the relevant managerial decisions. The considered approach is illustrated by the following scheme (figure 1).

In the drawing 1 reinforced line corresponds to such option of implementation of the operated process at which completely there are no deviations from balance. Real dynamics in the generalized view represents change of cycles of positive and negative deviations. If these deviations do not go beyond admissible limits (that is settle down in borders from - $\varepsilon$ to $+\varepsilon$ ), then and the condition of the operated process as it was already noted, it is possible to recognize satisfactory. 


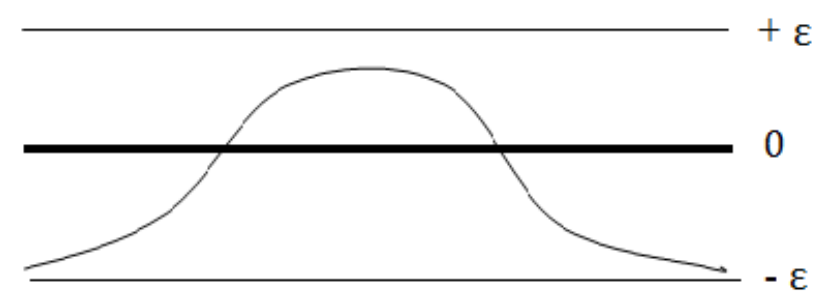

Fig. 1. Indicator tolerance from the calculated value of balance.

Calculation of balances / imbalances needs to be carried out separately for each of types of facilities of social infrastructure, having values for comfortable residence in the city:

- the number of school places;

- capacity of clinics;

- the number of places in hospitals, etc.

Formation of territorial balance (as well as identification of imbalances for their subsequent minimization and elimination) has to be provided at least at 2 levels:

1) at the level of the city in general;

2) at the level of certain areas.

In the conditions of megalopolises the second approach is especially important as the movement around the city to a facility of social infrastructure can take considerable time while necessary facilities of social infrastructure have to be available.

Comparison of indicators of need and providing with certain types of facilities of social infrastructure at the level of areas will allow to assess adequately a situation and to make the relevant managerial decisions on elimination of the available spatial disproportions (formation of the territorial balance covering, finally, all the territory of the city).

Separate task when planning urban development is determination of range $\pm \Delta \varepsilon$ within which the managerial situation is recognized as satisfactory and not demanding the correcting managerial influences. Besides, the size of an imbalance has to define urgency of measures of the city authorities for minimization and gradual elimination of territorial imbalances. Respectively, ranging of imbalances in size (deviation range between the actual level of security and requirement) is necessary. The following hierarchy of territorial balances in size (table 1) can be offered:

Table 1. Hierarchy of territorial balances.

\begin{tabular}{|c|l|}
\hline Imbalance $(\Delta)$ & \multicolumn{1}{c|}{ Characteristic of the imbalance and its rank } \\
\hline$\Delta \leq 2 \%$ & $\begin{array}{l}\text { The nominal imbalance with the lowest rank, has the lowest priority when } \\
\text { developing managerial decisions. Within the given deviation it is possible to } \\
\text { mention the existence of territorial balance as the deviation is not } \\
\text { considerable. }\end{array}$ \\
\hline $2 \% \geq \Delta \leq 10 \%$ & $\begin{array}{l}\text { The "standard" imbalance, has the rank, next on the increase, the sequence of } \\
\text { elimination is defined by the developed logical algorithm. }\end{array}$ \\
\hline $10 \% \geq \Delta \leq 25 \%$ & $\begin{array}{l}\text { The pre-critical imbalance, possesses the highest rank and prime priority of } \\
\text { elimination. }\end{array}$ \\
\hline$\Delta \geq 25 \%$ & $\begin{array}{l}\text { The critical imbalance, has the extra category rank and is the subject to } \\
\text { extraordinary elimination. }\end{array}$ \\
\hline
\end{tabular}

In this case the hierarchy is made up of the imbalances of four groups (ranks) from the lowest priority in the need of managerial influences to the extra category rank of the 
imbalance at which need of urgent, extraordinary actions for correction of a situation and minimization of the developed territorial imbalances is supposed. Certainly, the borders between ranks can be discussed and change in each case.

\section{Discussions}

Calculations of the territorial balances (imbalances) allow to determine the volumes of construction works, which need to be executed for the formation of the comfortable environment of activity. It allows to solve more serious problems of planning of activity of the investment and construction sphere of the megalopolis. It is actually possible to say that on the basis of calculations of the territorial imbalance and determination of volumes of construction of facilities of social infrastructure balance models of other order can be constructed.

So, the volumes of construction allow to determine the necessary volumes of material resources which will be required to implementation of plans for minimization and gradual elimination of territorial imbalances. The need for material resources (construction materials) can be estimated in comparison with the volume and structure of production of material resources in the region. The existence of an imbalance by results of comparison of requirement and the outputs will be about the existence or lack of a material imbalance. That allows to plan purchase terms, logistics and delivery periods of material resources, necessary for construction, which shortage will be found by results of formation of imbalances.

Also it is possible to estimate the need for the manpower of different profile and qualification on the basis of data on volumes of construction. The comparison of need for the manpower and its existence in the territory of the region can be also realized within balance model and formation of labor balance. The shortage of manpower revealed, when forming labour balance proves the need of attraction of manpower from other regions for the implementation of investment and construction projects.

The highest level of balance models in relation to the realization of the city-planning actions, connected with elimination of territorial imbalances is the model of financial balance. The required volumes of construction allow to estimate in less detail volumes of financial resources which have to be spent from means of the city budget and also the amount of funds of private investors who it is necessary to attract to formation of the comfortable environment of activity and sufficient level of providing the population with the facilities of social infrastructure.

\section{Conclusion}

The broad use of technologies of high-rise construction in the modern cities and megalopolises both within complex development of the whole areas, and within updating of the existing building, results in the need of improvement of managerial tools on planning of the city development. The special author's attention is paid to the questions of formation of the comfortable urban environment with proper providing the population with the facilities of social infrastructure (that is compliance of level of providing with the facilities of social infrastructure and the need for them). For performance of this task use of balance models is offered and the concept of territorial balance which is understood as compliance of structure and dimensional indicators of the facilities of social infrastructure within a concrete spatial fragment of the territory of the city of the established requirement is entered. The formula of calculation of level of providing with the facilities of social 
infrastructure which is applied to calculation of level of security separately by each type of facilities is offered.

Separately it is necessary to notice that use of these tools is especially relevant in a situation when within the developed building investment and construction projects of multy-storied construction which can lead to considerable change of a ratio of the residential real estate and objects of social infrastructure on the concrete territory are implemented. The discrepancy of the actual level of security in objects of social infrastructure and requirement leads to formation of a territorial imbalance. The formula of calculation of a territorial imbalance both in relative, and in absolute expression is offered and proved that allows to determine finally the construction volumes necessary for elimination of territorial imbalances.

The imbalance is of great importance for planning and realization of managerial influences from the executive authorities, operating the city development. For the determination of urgency of realization of actions for construction of the facilities of social infrastructure it is offered to range imbalances in size, having determined the deviation size from balance which is so insignificant that does not demand managerial influences (it can be characterized as balance) and also the groups of the imbalances differing in urgency managerial influences, directed to the decrease and elimination of the revealed imbalance.

One can estimate the necessary volumes of construction of the facilities of social infrastructure, as well as material, labour and financial resources, which will be required for the elimination of territorial imbalances, on the basis of data on territorial imbalances.

\section{References}

1. J. Burton, Worldatlas (2017) https://www.worldatlas.com/articles/cities-with-the-mostskyscrapers-in-the-world.html

2. W.S. Mathers, Location https:// http://www.realmarkits.com/realestate/locationvalue.php (2013)

3. G. W. McCarthy, G. K. Ingram, S.A. Moody, Land and the City (2014)

4. V. Verkhie, The value and price of land, 40-62 (2017) https:// https://www.eolss.net/sample-chapters/C19/E1-05-03-03.pdf

5. T. Gwartney, Estimating Land Values (2018) http://www.henrygeorge.org/ted.htm

6. M. Lomedico, E.M. Bartels, A Structured Analytical Approach to Understanding and Acting in Urban Environments (2015)

7. A. Perdicoulis, The city as a system, 16 (2013)

8. G. Gardner, State of the World. The City: A System of Systems, 27-44 (2016)

9. W. Leontief, The Structure of American Economy (1919-1929)

10. W. Leontief, Input-Output Economics (1988).

11. P. Krylov, Journal Problems in Economics. The Balance Method of Planning, 11 (1968)

12. N.G. Verstina, T.N. Kisel, N.N. Taskaeva, E.M. Akimova, A.V. Fedosina, International Journal of Applied Engineering Research Managing to provide the building industry of a megalopolis with material resources on the basis of balance method (2015)

13. T.N. Kisel, M.I. Bunegin, E.M. Akimova, Economics and Entrepreneurship. Approach to planning the development of territories in conditions of ensuring a balanced development of the city, 6-3 (59-3), 291-295 (2015)

14. M.I. Bunegin, Kazan science, 8, 34-36 (2014)

15. A. Tlidi, International Journal of Economics and Financial Issues, 3, 932-937 (2013) 